\title{
Pantoea agglomerans: ¿un nuevo patógeno en la unidad de cuidados intensivos neonatales?
}

\author{
Pantoea agglomerans: a new pathogen at the neonatal intensive care unit?
}

\author{
Dr. Antonio Segado-Arenas ${ }^{a}$, Dra. Almudena Alonso-Ojembarrena ${ }^{a}$, Dr. Simón P. Lubián-López y \\ Dra. Ana M. García-Tapia
}

\begin{abstract}
RESUMEN
La sepsis tardía es especialmente frecuente en los recién nacidos pretérmino, y los bacilos gramnegativos son responsables de los casos más graves con una elevada mortalidad asociada. Pantoea agglomerans es un bacilo gramnegativo que pocas veces se ha descrito como patógeno en el ser humano, menos aún en el recién nacido. Se presenta el caso clínico de un recién nacido pretérmino que sufrió una sepsis fulminante por Pantoea agglomerans en una unidad de cuidados intensivos neonatales. Hasta la fecha no se ha descrito ningún caso de sepsis por $P$. agglomerans en esta población en España.

Palabras clave: neonato, Pantoea agglomerans, sepsis tardía.
\end{abstract}

\section{SUMMARY}

Late-onset sepsis is very frequent among preterm infants and cases due to Gram negative pathogens have elevated morbidity and mortality. Pantoea agglomerans is a Gram negative organism which has been rarely reported causing disease in humans. We present a case of $P$. agglomerans late-onset fulminant sepsis in a preterm newborn at a neonatal intensive care unit. Up to date none P. agglomerans sepsis has been reported among this population in our country.

Keywords: newborn, Pantoea agglomerans, late-onset sepsis.

http:/ / dx.doi.org/10.5546/aap.2012.e77

\section{INTRODUCCIÓN}

Pantoea agglomerans es una enterobacteria aislada frecuentemente en plantas, frutas y vegetales, pero que también se ha encontrado en heces humanas y de animales. ${ }^{1}$ Como patógeno, se ha descrito clásicamente como causante de infecciones localizadas (sinovitis, artritis) postraumatismo con plantas. ${ }^{2,3}$ Los primeros casos de sepsis en recién nacidos (RN) y lactantes fueron secundarios

a. Sección de Neonatología. Unidad de Gestión Clínica de Pediatría.

b. Servicio de Microbiología. Secretaría de Neonatología. Hospital Universitario Puerta del Mar.

Cádiz. España.

Correspondencia:

Dr. Antonio Segado Arenas: a.segadoarenas@gmail.com

Conflicto de intereses: Ninguno que declarar.

Recibido: 30-1-2012

Aceptado: 28-3-2012 a contaminación de fluidos intravenosos. ${ }^{4,5} \mathrm{Si}$ se eliminan las sepsis secundarias a contaminación de nutrición parenteral (NP), se han descripto sólo 11 casos de sepsis neonatal por P. agglomerans, ninguno en España. ${ }^{6-9}$

\section{CASO CLÍNICO}

Recién nacida pretérmino de 32 semanas y dos días de gestación, fruto de gestación gemelar $\left(2^{\mathrm{a}}\right.$ gemela). Oligoamnios y crecimiento intrauterino retardado desde la semana 20 de gestación. Serologías para Toxoplasma, citomegalovirus, rubéola y herpes virus negativas, marcadores hepáticos negativos. Nace mediante cesárea electiva por detectarse en ecografía Doppler flujos arteriales alterados en el segundo gemelar. Apgar de 5 y 7 al primer minuto y a los cinco minutos, respectivamente. Peso de RN: 750 g.

Permaneció respiratoriamente estable sin necesidad de soporte respiratorio u oxigenoterapia.

Requirió catéter venoso umbilical, retirado a los 7 días de vida (cultivo del catéter negativo, se sustituyó por catéter epicutáneo) y de NP. Antibioterapia con ampicilina y gentamicina y profilaxis de candidiasis con fluconazol desde el nacimiento. A los 8 días de vida presentó sepsis tardía, con fiebre, elevación de reactantes de fase aguda y crisis de apnea-bradicardia, por lo que se inició tratamiento con vancomicina. En el hemocultivo se aisló Staphylococcus coagulasa negativo.

A los 19 días de vida comenzó con mala perfusión periférica (piel reticulada, frialdad acra, palidez, pulsos filiformes), dificultad respiratoria (polipnea, respiración superficial, tiraje subcostal) con necesidad creciente de oxigenoterapia para mantener una adecuada saturación de oxígeno, taquicardia (de hasta 195 latidos por minuto) y oligoanuria. Fue intubada y conectada a ventilación mecánica. Además se inició soporte hemodinámico con expansiones de volumen repetidas (salino al 0,9\% y plasma fresco congelado) y tratamiento inotrópico con dopamina (hasta $15 \mu \mathrm{g} / \mathrm{kg} / \mathrm{min}$ ) y adrenalina (hasta $0,2 \mu \mathrm{g} / \mathrm{kg} /$ $\mathrm{min}$ ), estableciéndose un shock refractario a catecolaminas. Se añadió meropenem al tratamiento 
antibiótico con vancomicina. La gasometría reveló una acidosis metabólica ( $\mathrm{pH}$ : 7,22 con ácido láctico de 3,4 mmol/L). En control analítico presentó una coagulopatía grave con afectación de las 2 vías de coagulación (INR: 2,1; actividad de protrombina del $34 \%$; tiempo de tromboplastina parcial activada: $42,70 \mathrm{seg}$ ) y elevación de los reactantes de fase aguda (proteína $\mathrm{C}$ reactiva [PC-R] de $198 \mathrm{mg} / \mathrm{L}$ ).

A pesar de todas las medidas de soporte, el estado hemodinámico empeoró, hubo shock refractario instaurado y se añadió al cuadro una hemorragia pulmonar aguda, con hipoxemia grave a pesar de la intensificación del soporte respiratorio. Obitó a las $4 \mathrm{~h}$ de iniciado el cuadro y no se realizó estudio necrópsico.

En el hemocultivo extraído se aisló una cepa salvaje de Pantoea agglomerans, sensible a betalactámicos, quinolonas, gentamicina, amikacina y meropenem, con ausencia de mutaciones en topoisomerasas (Figura 1). El tiempo de crecimiento del germen fue de $7 \mathrm{~h}$ y $15 \mathrm{~min}$. El cultivo de la NP fue negativo.

\section{DISCUSIÓN}

En un informe reciente del National Institute of Child Health and Human Development Neonatal Research Network, se describe una incidencia de sepsis tardía en RNPT de MBPN de hasta el 25\%, con una mortalidad de hasta el 18\%. Los bacilos gramnegativos fueron los segundos en frecuencia (17\%) tras Staphylococcus coagulasa-negativo, pero fueron los que más mortalidad asociaron $(36,2 \%) .{ }^{10}$

Pantoea agglomerans (antes conocida como Enterobacter agglomerans y antes como Erwinia agglomerans) es una bacteria gramnegativa de la familia Enterobacteriaceae descrita ocasionalmente como patógeno en seres humanos; se lo considera un germen oportunista. Se han publicado casos de infección localizada por pinchazos con espinas de plantas o astillas, ${ }^{2,3}$ así como casos de peritonitis en pacientes portadores de dispositivos invasivos. ${ }^{11-13}$ En población pediátrica se ha descrito un brote de sepsis por P. agglomerans secundario a contaminación de catéteres intravenosos en una sala de observación de urgencias, aunque no se trató de cuadros graves. ${ }^{14}$

Figura 1. A la izquierda colonias de P. agglomerans, que presentan la típica pigmentación amarilla en un medio de agar sangre. Observése la diferencia de pigmentación con las colonias de otra especie de enterobacteria, en la imagen de la derecha.

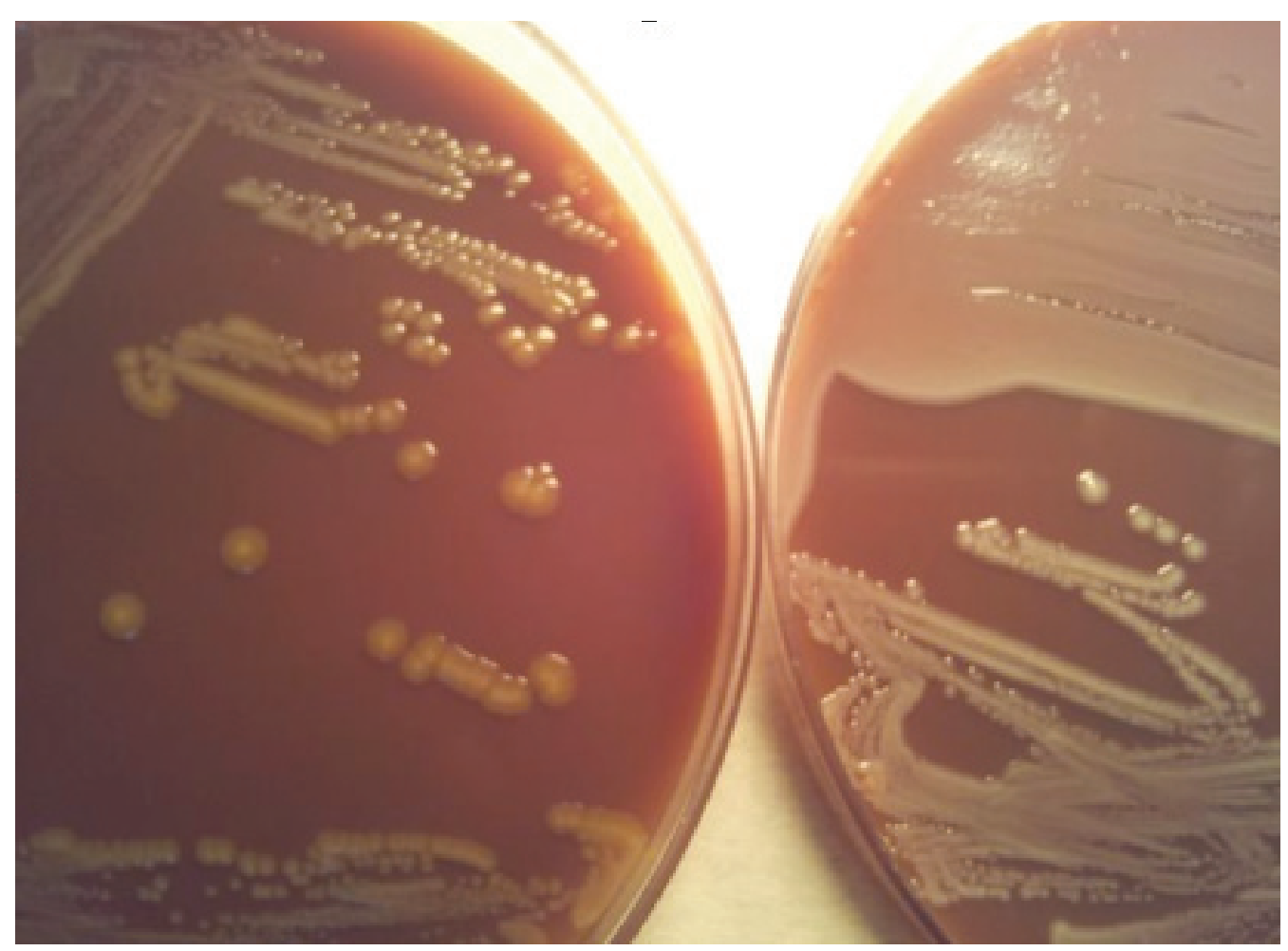


En población neonatal se han descrito un total de 18 casos de sepsis tardía por P. agglomerans hasta la fecha. En estas series llama la atención la elevada mortalidad $(10 / 18)$ y la refractariedad al tratamiento antibiótico, a pesar de haberse indicado con antibióticos a los que el germen era sensible. 5,9 En la serie de van Rostenberghe et al., con 8 pacientes que sufrieron sepsis por $P$. agglomerans secundaria a contaminación de $\mathrm{NP}$, hubo una elevada mortalidad $(7 / 8)$ con mala respuesta al tratamiento antibiótico. Se postuló que la mala evolución se debió a la gran virulencia debido a la inoculación directa del germen en el torrente sanguíneo. ${ }^{5}$ En cuanto a los casos de sepsis neonatal tardía esporádica, hay descritos 8 casos con una evolución muy diferente. Aly et al. presentan 5 casos con buena evolución y una supervivencia del $100 \%$. De ellos, ninguno presentó shock ni coagulación intravascular diseminada (CID), además tuvieron valores de PC-R por debajo de 100 $\mathrm{mg} / \mathrm{L}(3,4-96 \mathrm{mg} / \mathrm{L}) .{ }^{8}$ En cambio, Bergman et al. describen 3 casos con una mortalidad del $100 \%$. Los pacientes de esta serie sufrieron shock, CID y presentaron PC-R más elevadas que los de la serie de Aly et al. (143-334 mg/L). Todos los pacientes fallecieron a pesar del tratamiento antibiótico. ${ }^{9}$ Nuestra paciente presentó shock refractario a fármacos, CID y un valor de PC-R inicial de 198 $\mathrm{mg} / \mathrm{L}$, además de hemorragia pulmonar.

Parece claro que en los casos secundarios a contaminación de productos intravenosos, la mala evolución se debe a la gran carga microbiana a la que son sometidos estos pacientes, con mala respuesta a un tratamiento antibiótico correcto. En cuanto a la disparidad en la evolución de los casos esporádicos no está clara su causa. Una teoría que puede explicar la mala evolución es que la sepsis tenga su origen en un foco localizado de infección (absceso, trombo, catéter intravascular) que perpetúe la situación de septicemia. Nuestro caso, al igual que los 3 descritos por Bergman et al., presentó características de una sepsis ya avanzada en el momento del diagnóstico (CID, shock irreversible y PC-R elevada) mientras que los pacientes de Aly et al. (todos ellos con buena evolución) pudieron haber sido diagnosticados en una fase mucho más precoz de la enfermedad, como parecen indicar la ausencia de hipotensión y CID, y los valores más bajos de PC-R.

En cuanto a la hemorragia pulmonar se ha descrito previamente en $5 / 18$ pacientes. Esto puede ser explicado por la gravedad de las alteraciones en la coagulación y por el trofismo pulmonar que ha mostrado P. agglomerans en algunos casos, habiéndose descrito neumonía concomitante con sepsis en $5 / 18$ casos.

\section{CONCLUSIONES}

La sepsis neonatal por P. agglomerans es un cuadro infrecuente, pero que reviste mucha gravedad, con una elevada mortalidad asociada. A pesar de tener un perfil de sensibilidad antibiótica amplio, la respuesta a antibióticos es, en muchos casos, deficiente. Corresponde hacer hincapié en las medidas de prevención: limitar los días de uso de NP y cuidar su esterilidad, la máxima asepsia en la manipulación de accesos intravenosos, y el uso criterioso de antibióticos.

\section{BIBLIOGRAFÍA}

1. Andersson AM, Weiss N, Rainey F, Salkinoja-Salonen MS. Dust-borne bacteria in animal sheds, schools and children's day care centres. J Appl Microbiol 1999;86:622-34.

2. Kratz A, Greenberg D, Barki Y, Cohen, Lifshitz M. Pantoea agglomerans as a cause of septic arthritis after palm tree thorn injury; case report and literature review. Arch Dis Child 2003;88:542-4.

3. De Champs C, Le Seaux S, Dubost JJ, Boisgard S, et al. Isolation of Pantoea agglomerans in two cases of septic monoarthritis after plant thorn and wood sliver injuries. J Clin Microbiol 2000;38:460-1.

4. Matsaniotis NS, Syriopoulou VP, Theodoridou MC, Tzanetou KG. Enterobacter sepsis in infants and children due to contaminated intravenous fluids. Infect Control 1984;5:471-7.

5. Van Rostenberghe H, Noraida R, Wan Pauzi WI, Habsah $\mathrm{H}$, et al. The clinical picture of neonatal infection with Pantoea species. Jpn J Infect Dis 2006;59:120-1.

6. Cruz AT, Cazacu AC, Allen CH. Pantoea agglomerans, a plant pathogen causing human disease. J Clin Microbiol 2007;45:1989-92.

7. Lalas KM, Erichsen D. Sporadic Pantoea agglomerans bacteremia in a near-term female: case report and review of literature. Jpn J Infect Dis 2010;63:290-1.

8. Aly NY, Salmeen HN, Abo Lila RA, Nagaraja PA. Pantoea agglomerans bloodstream infection in preterm neonates. Med Princ Pract 2008;17:500-3.

9. Bergman KA, Arends JP, Scholvinck EH. Pantoea agglomerans septicemia in three newborn infants. Pediatr Infect Dis J 2007;26:453-4.

10. Downey LC, Smith PB, Benjamin DK Jr. Risk factors and prevention of late-onset sepsis in premature infants. Early Hum Dev 2010;86:7-12.

11. Kahveci A, Asicioglu E, Tigen E, Ari E, Z et al. Unusual causes of peritonitis in a peritoneal dialysis patient: Alcaligenes faecalis and Pantoea agglomerans. Ann Clin Microbiol Antimicrob 2010;10:12-4.

12. Lim PS, Chen SL, Tsai CY, Pai MA. Pantoea peritonitis in a patient receiving chronic ambulatory peritoneal dialysis. Nephrology 2006;11:97-9.

13. Magnette C, Tintillier M, Horlait G, Cuvelier C, Pochet JM. Severe peritonitis due to Pantoea agglomerans in a CCPD patient. Perit Dial Int 2008;28:207-8.

14. Bicudo EL, Macedo VO, Carrara MA, Castro FF, Rage RI. Nosocomial outbreak of Pantoea agglomerans in a pediatric urgent care center. Braz J Infect Dis 2007;11:281-4. 